\title{
An Intelligent Fault Detection Model for Fault Detection in Photovoltaic Systems
}

\author{
Barun Basnet $\mathbb{D}$, Hyunjun Chun, and Junho Bang $\mathbb{D}$ \\ Dept. of IT Applied System Engineering, Jeonbuk National University, Republic of Korea \\ Correspondence should be addressed to Junho Bang; jhbang@jbnu.ac.kr
}

Received 11 February 2020; Revised 12 May 2020; Accepted 19 May 2020; Published 9 June 2020

Academic Editor: Manuel Aleixandre

Copyright (C) 2020 Barun Basnet et al. This is an open access article distributed under the Creative Commons Attribution License, which permits unrestricted use, distribution, and reproduction in any medium, provided the original work is properly cited.

\begin{abstract}
Effective fault diagnosis in a PV system requires understanding the behavior of the current/voltage (I/V) parameters in different environmental conditions. Especially during the winter season, I/V characters of certain faulty states in a PV system closely resemble that of a normal state. Therefore, a normal fault detection model can falsely predict a well-operating PV system as a faulty state and vice versa. In this paper, an intelligent fault diagnosis model is proposed for the fault detection and classification in PV systems. For the experimental verification, various fault state and normal state datasets are collected during the winter season under wide environmental conditions. The collected datasets are normalized and preprocessed using several data-mining techniques and then fed into a probabilistic neural network (PNN). The PNN model will be trained with the historical data to predict and classify faults when new data is fetched in it. The trained model showed better performance in prediction accuracy when compared with other classification methods in machine learning.
\end{abstract}

\section{Introduction}

Fault detection and timely troubleshooting are essential for the optimum performance in any power generation system, including photovoltaic (PV) systems. In particular, the goal for any commercial power-producing house is maximizing power production, minimizing energy loss and maintenance cost, and the safe operation of the facility. Since PV systems are subject to various faults and failures, early detection of such faults and failures is very crucial for achieving the goal [1-3]. The US National Electric Code requires the installation of OCPD (Overcurrent Protection Device) and GFDI (Ground Fault Detection Interrupters) in PV installations for protection against certain faults. However, the Bakersfield Fire case, 2009, and Mount Holly, 2011, show the inability of these devices to detect the fault in those particular scenarios [4]. Faults in a PV system can arise from either physical, environmental, or electrical conditions $[5,6]$.

A wide range of technologies exist for PV array fault detection, and also extensive studies have been done in the area to offer possible solutions [7]. The two most important parameters in determining the performance in a PV system are current and voltage. A simple current-voltage analysis method was proposed where the electrical signature of each faulty modules and array was fixed by considering the deformations induced on the I/V curves [8]. Another study shows the use of infrared thermography where electrical and thermal models of a PV system were combined for extracting quantitative information of a mismatch fault [9]. Similar studies show the application of aerial infrared thermography for detection of the damage on the PV blocks [10] and an onfield infrared thermography-sensing technique for PV system efficiency assessment [11]. Likewise, reflectometry methods have also been used for fault detection in PV systems. A time domain reflectometry (TDR) method was used to detect short circuit and insulation defects $[12,13]$, and recently, a spread spectrum TDR (SSTDR) method was investigated to detect ground faults and aging-related impedance variations in a PV system [14]. In addition to that, the application of wavelet decomposition techniques for 


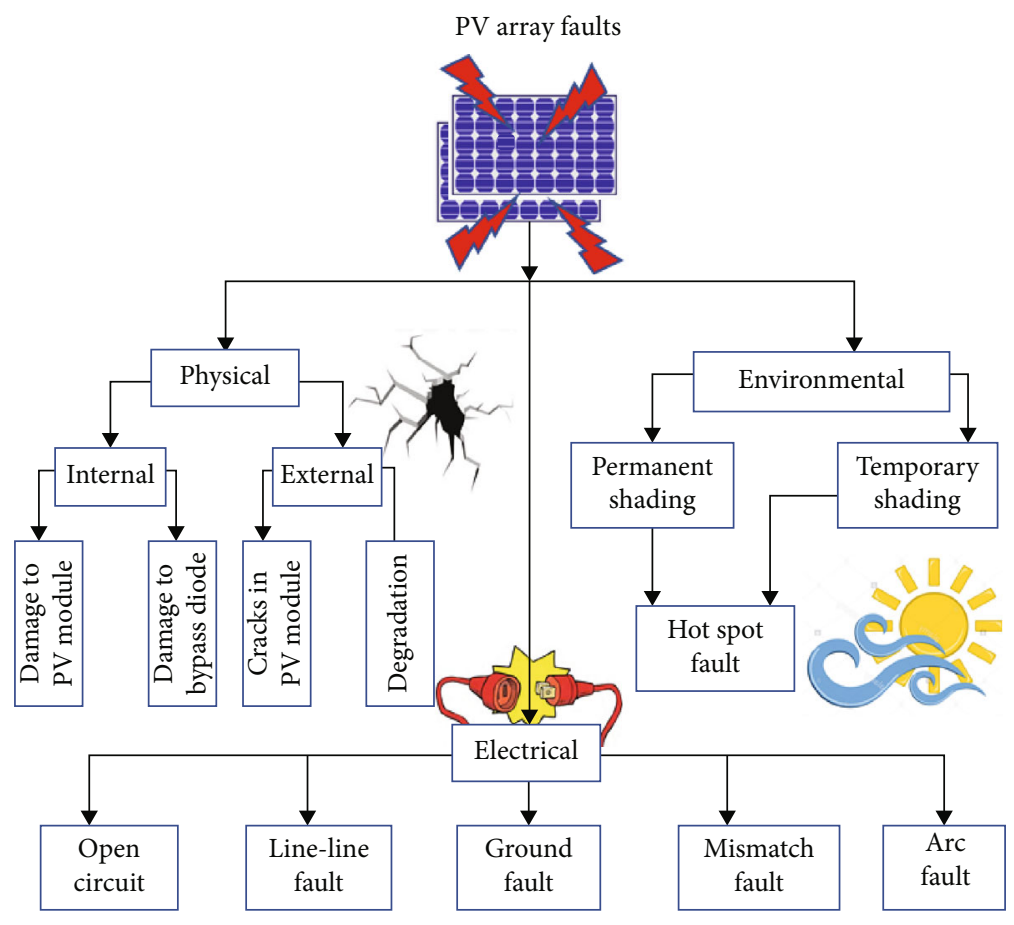

FIgURE 1: Classification of PV array faults.

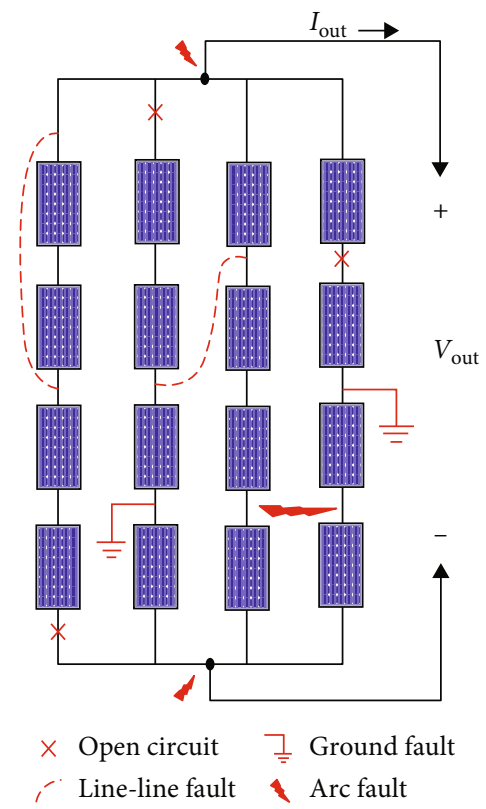

Figure 2: Main types of electrical faults.

detecting arc faults [15-17] and multiresolution signal decomposition for detecting line-line faults $[18,19]$ are also found in the literature. A recent article has provided a comprehensive study on several advanced fault detection approaches in PV systems. The study has divided fault detection approaches into model-based difference measurement (MBDM), real-time difference measurement (RDM), output signal analysis (OSM), and machine learning techniques
(MLT). It has also done critical comparisons of these advanced techniques with conventional methods providing their pros and cons [20].

Nowadays, most of the PV systems are built with a monitoring system and have a database constantly backed with huge historical data [21]. Artificial Intelligence (AI) methods are data-based, and with the availability of big data in PV systems, studies in this area seem to be in the momentum. In particular, machine learning- (ML-) based algorithms and techniques are proposed [22-25], where the model is trained with historical data to predict and classify faults. A recent study reports the application of thermography and ML techniques for fault classification in PV modules [26]. The study has adopted a texture feature analysis to study the features of various fault panel thermal images, and the developed algorithm was trained with 93.4\% accuracy. Another study reports the application of ML techniques for fault detection, classification, and localization in PV systems. The study claims the development of the algorithm with the prediction accuracy of $100 \%$ [27]. Likewise, another study utilizes a waveletbased approach and radial basis function networks (RBFN) to detect short circuit and open circuit faults in the inverter [28]. Their work presents $100 \%$ training efficiency and $97 \%$ testing efficiency when tested in a $1 \mathrm{~kW}$ single-phase stand-alone PV system.

The performance of a trained model for a PV system using ML techniques can greatly vary if new data is fetched from a different environmental condition, especially data from the winter season. The irradiation level in the winter is substantially lower than that in the summer, and studies have shown faults occurring in such lower irradiation levels 


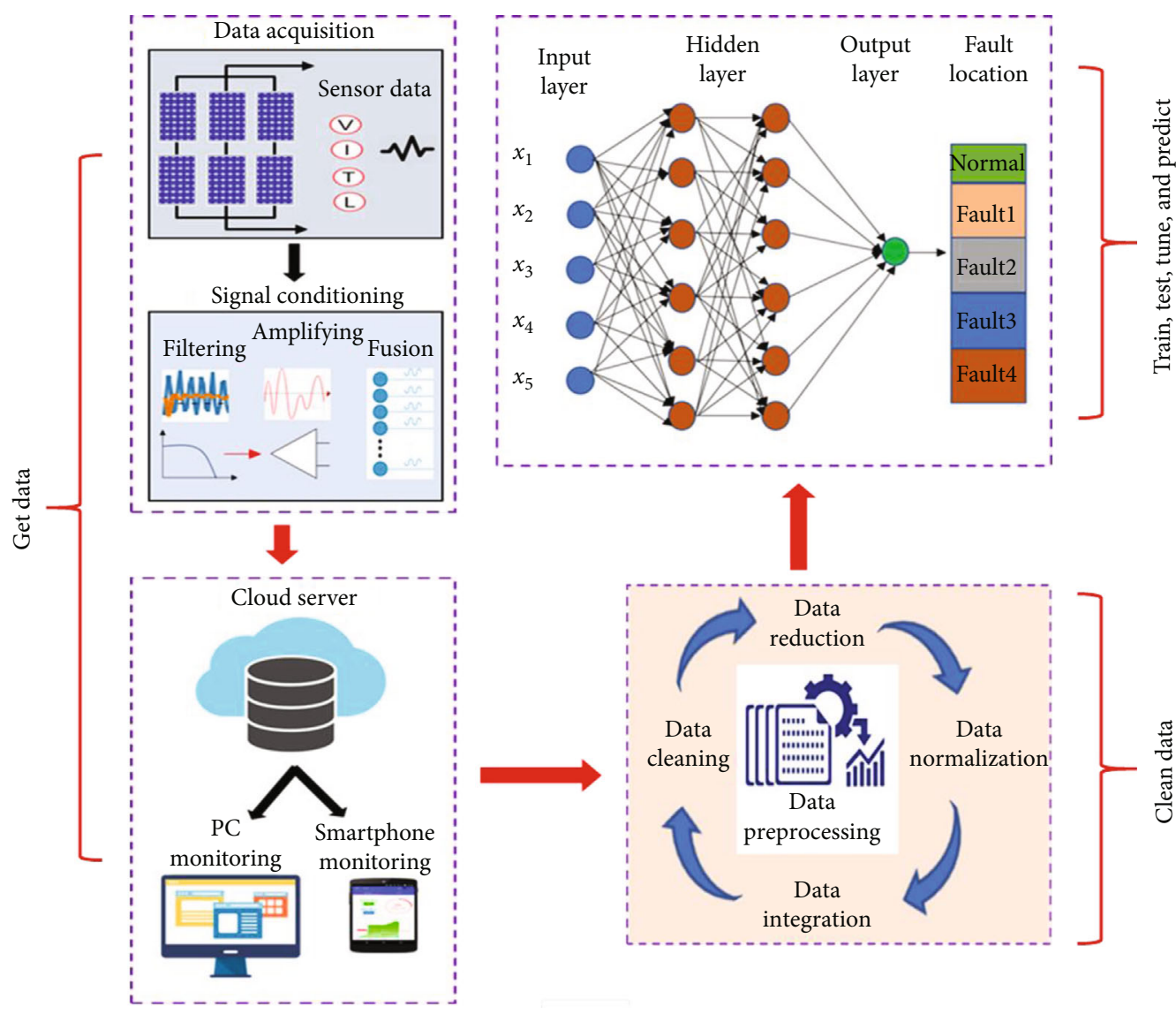

FIGURE 3: Proposed fault diagnosis system architecture.

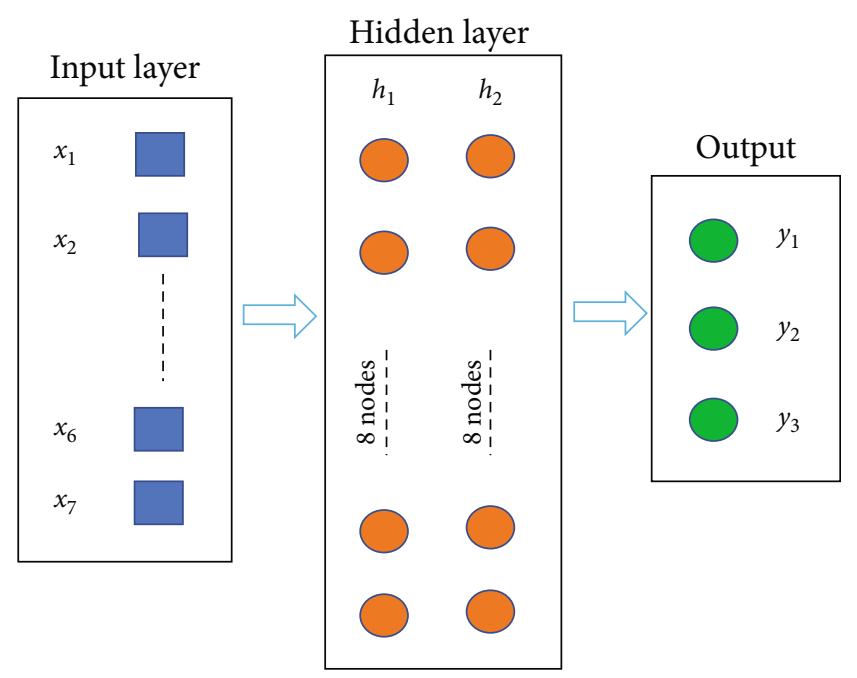

FIGURE 4: Multilayer perceptron used for the model.

have higher chances of remaining undetected [29, 30]. Such undetected faults can cause a significant amount of power losses and degradation of the quality of the panel or even lead to deterioration of panels. We propose an intelligent fault diagnosis model for detecting faulty modules and further classifying the fault type that is applicable in all environmental conditions. The model uses the multilayer perceptron
(MLP) and follows the supervised learning approach. It is robustly trained with historical data of different faulty and normal states in different environmental conditions especially focusing on winter. The data was collected from a $1.8 \mathrm{~kW}$ grid-connected PV system located at Jeollabuk-do province of South Korea.

The rest of the paper is organized as follows. Section 2 introduces the overview of PV system faults. Section 3 describes the whole system architecture of the fault diagnosis model. Section 4 presents experimental results, compares the model with existing classification methods, and discusses other relevant issues. Finally, Section 5 summarizes and concludes the article.

\section{Overview of PV System Faults}

The classification of faults occurring in a PV system can be categorized from different aspects. We classify such faults into three types: physical, environmental, and electrical $[2,3]$. However, the classification of faults can also be made on other bases, e.g., location and structure [1].

Physical faults can be internal or external and generally include damage, cracks, and degradation in PV modules. Also, PV system failures are caused by the aging effect which is also a physical phenomenon.

Environmental faults include soiling and dust accumulation, bird drops, and temporary shading. Permanent 


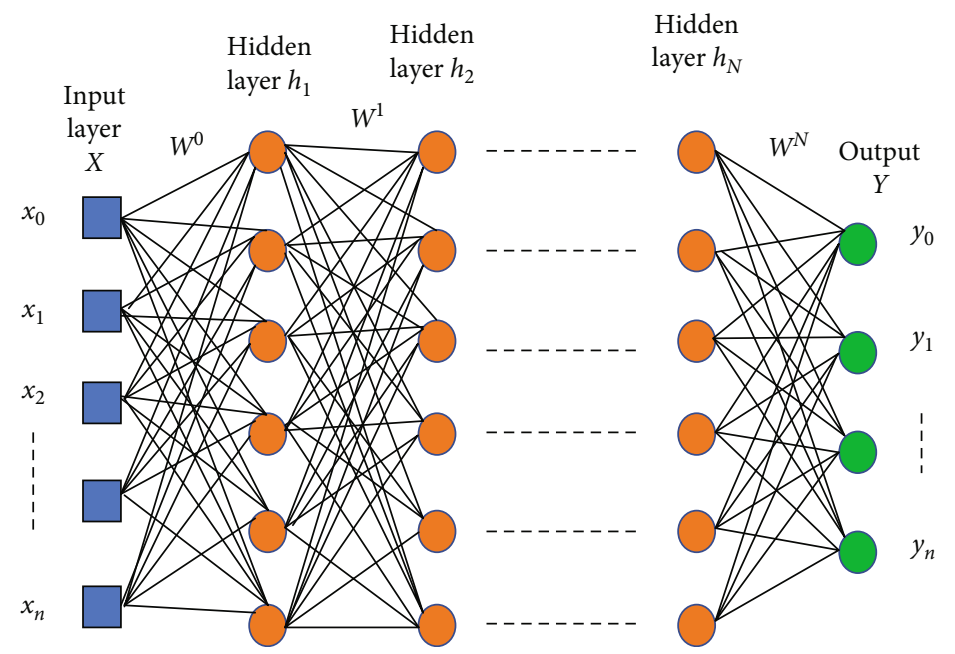

FIGURE 5: Feedforward multilayer perceptron.

environmental faults include permanent shading due to the poor choice of installment location. Hotspot faults in the PV modules can be caused by both permanent and temporary shading. Lastly, electrical faults include open circuit, line-line, and ground faults, either in PV modules, arrays, or in the whole systems. Open circuit faults are caused by the disconnection of wires in single or multiple branches of a PV circuit.

Line-line faults are created by unintentional low impedance current path in a PV array. Ground faults are similar to line-line faults; however, the low impedance path is from current-carrying conductors to ground/earth. Figure 1 shows the classification of PV array faults, whereas Figure 2 shows the main types of electrical faults in PV systems.

A PV module can be modeled electrically with a one diode or two diode model [18]. However, modeling a real PV system is very complex because electrical parameters vary largely between PV systems due to variation in the construction of PV modules (dimension, material, and ground connection), site, and physical layout [27]. Especially in largescale power generation systems, modeling a system comes with the special technical challenge. In this study, we have limited our work to detect only electrical faults.

\section{Proposed System Architecture}

This chapter provides detailed explanation of the several steps that constitute the proposed fault diagnosis system architecture. Figure 3 shows the block diagram and the flow of each step in the proposed architecture.

3.1. Data Acquisition. This is the first layer of the system architecture. For building the model, we acquired the current, voltage, irradiation level, and temperature data from respective sensors attached to the PV array. The sensors operate at the $5 \mathrm{~V}$ level, while the PV module used in this study has an open circuit voltage $\left(V_{o c}\right)$ of $39 \mathrm{~V}$ and short circuit current $\left(I_{\mathrm{sc}}\right)$ specification of $9 \mathrm{~A}$. Active analog filters were used to remove noise levels that could get injected into
TABle 1: Parameters used in the MLP.

\begin{tabular}{|c|c|}
\hline Parameters & Values \\
\hline Algorithm & Stochastic gradient descent \\
\hline Activation function & $\begin{array}{l}\text { ReLU (input, hidden layers) } \\
\text { Softmax (output layer) }\end{array}$ \\
\hline Layer & $\begin{array}{l}3 \text { hidden layers with } 8 \text { units each } \\
3 \text { units at the output }\end{array}$ \\
\hline Loss function & Categorical crossentropy \\
\hline Optimizer & Adam \\
\hline Data split & Train: $80 \%$, test: $20 \%$ \\
\hline Batch size & 5 (with 200 epochs) \\
\hline Tuning & $k$-Fold crossvalidation, dropout \\
\hline
\end{tabular}

current and voltage sensors from the PV panel. The irradiation level data was collected using a commercial lux meter (LX1330B) with a 0.01 to 200 klux range and error rate of $\pm 2 \%$. Temperature data was collected from the sensor attached to the modules. The difference between ambient temperature and panel temperature was in the range of 1 to 7 degree Celsius $\left({ }^{\circ} \mathrm{C}\right)$. The input data fetched for training the model is the average temperature measured from each module. The dataset consists of data collected in summer and winter in all possible environmental conditions. The collected data was backed in the local server as well as the cloud server.

3.2. Data Preprocessing. Data preprocessing is the second layer in the proposed system architecture. It consists of all the actions taken before the data inputs are fetched to the model for extracting features. Figure 4 shows the functional block of the multilayer perceptron model used in this study. In order to create the fault detection model, seven PV data features are selected as the input attributes for the input layer.

$x_{1}$ is the current (A) in branch 1 of the PV system, $x_{2}$ is the current $(\mathrm{A})$ in branch 2 of the PV system, $x_{3}$ is the voltage (V) in branch 1 of the PV system, $x_{4}$ is the voltage 
TABLe 2: Data collected of the PV system in different states under different environmental conditions.

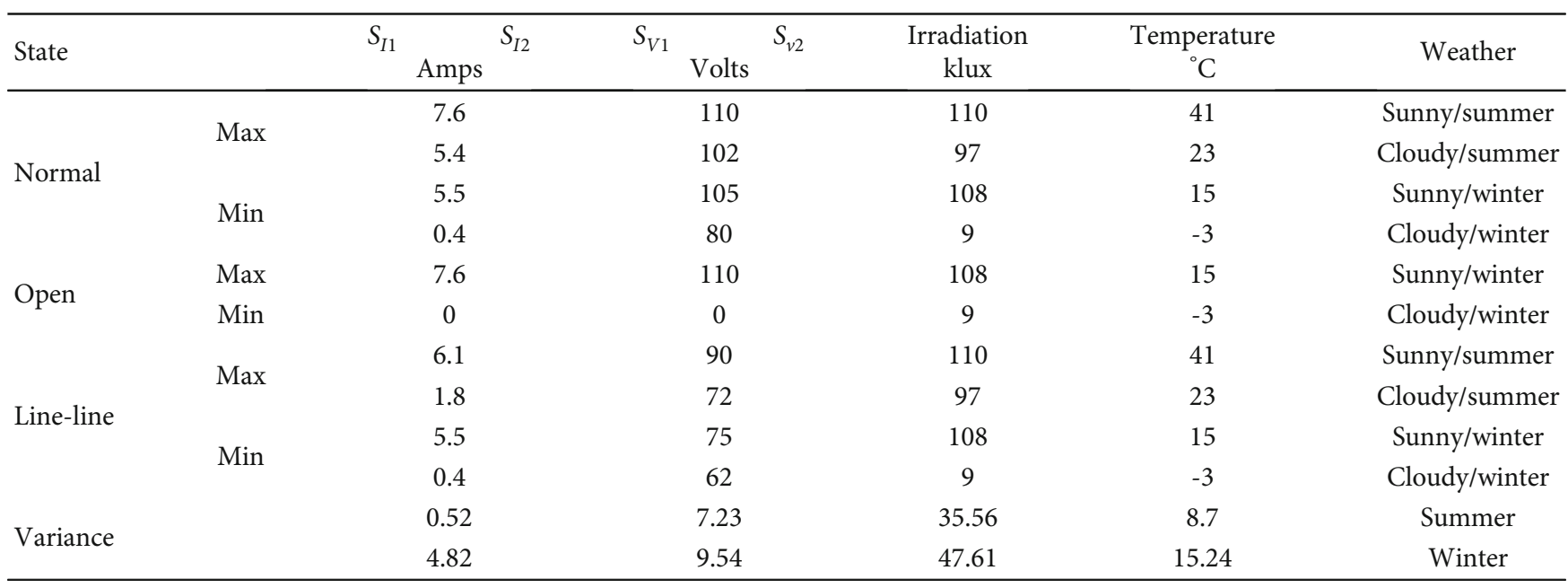

TABLE 3: Specification of the PV system.

\begin{tabular}{lc}
\hline Parameters & Values \\
\hline Maximum power $\left(P_{\max }\right)$ & $1.8 \mathrm{~kW}$ \\
Short circuit current $\left(I_{\mathrm{sc}}\right)$ & $9 \mathrm{~A}$ \\
Open circuit voltage $\left(V_{\mathrm{oc}}\right)$ & $39 \mathrm{~V}$ \\
Parallel connection & 2 lines \\
Serial connection & 3 panels \\
Connection type & Grid-connected \\
\hline
\end{tabular}

(V) in branch 2 of the PV system, $x_{5}$ is the irradiation level (klux), $x_{6}$ is the average temperature $\left({ }^{\circ} \mathrm{C}\right)$ from each module, and $x_{7}$ is the weather condition (sunny, snowy, cloudy, and rainy).

Among the input data, $x_{7}$ is of a categorical nature; thus, it is encoded to a suitable numerical data. The weather condition "sunny" was encoded to 1 and the rest ("snowy," "cloudy," and "rainy") were encoded to 0 as data collected in those environmental conditions showed quite a similar feature. After that, all the input data were normalized as follows:

$$
z=\frac{x-u}{s}
$$

where $z$ is the standard score of sample $x, u$ is the mean of the training samples, and $s$ is the standard deviation of the training samples. The whole dataset was split into the training set and the test set with the ratio of $8: 2$.

3.3. Multilayer Perceptron and Feature Extraction. A multilayer perceptron (MLP) or probabilistic neural network (PNN) is a nonlinear learning algorithm in ML and is widely applied in both supervised and unsupervised learning. However, most of its application is found in the classification problem of supervised learning.

$$
\Phi_{i j}(y)=\frac{1}{(2 \pi)^{1 / 2} \omega^{d}} \frac{1}{d} \sum_{1}^{d} e^{-\frac{\left(y-y_{i j}\right)\left(y-y_{i j}\right)^{T}}{\omega^{2}}} .
$$

Here, $\Phi_{i j}(y)$ is the probability density function of input vector $y, d$ is the total category number of training samples, $y_{i j}$ is the $j^{\text {th }}$ training center of the $i^{\text {th }}$ type of samples, and $\omega$ is the smoothing factor [23]. Figure 5 shows a feedforward multilayer perceptron.

Assuming that we used an input layer with $n_{0}$ neurons, input layer $X$ can be given as

$$
X=\left(x_{0}, x_{1}, \cdots, x_{n}\right)
$$

For the feature extraction, the hidden layer is designed with two layers: $h_{1}$ being the first hidden layer and $h_{2}$ being the second hidden layer. Each of the input dimensions $\left(x_{1}\right.$ to $\left.x_{7}\right)$ is fed to $h_{1}$, and then, the output from $h_{1}$ goes to $h_{2}$. The outputs $h_{i}^{j}$ of neurons in the hidden layers are computed as

$$
h_{i}^{j}=f\left(\sum_{k=1}^{n_{i-1}} W_{k, j}^{i-1} h_{i-1}^{k}\right), \quad i=2, \cdots, N, \bigotimes j=1, \cdots, n_{i},
$$

where $W_{k, j}^{i-1}$ is the weight between the neuron $k$ in the hidden layer I and neuron $j$ in the hidden layer $+1, n_{i}$ is the number of the neurons in the $i^{\text {th }}$ hidden layer. Both of the hidden layers use uniform distribution as the kernel initializer for initializing the weights in the network. Also, we chose ReLU (Rectified Linear Unit) as the activation function because of its several advantages in nonlinear datasets with multiple dimensions [31]. ReLU is given as

$$
y=\max (0, x)
$$

The output layer consists of three layers: $y_{1}, y_{2}$, and $y_{3}$. The network outputs are computed as

$$
y_{i}=f\left(\sum_{k=1}^{n_{N}} W_{k, j}^{N} h_{N}^{k}\right) \text {, }
$$




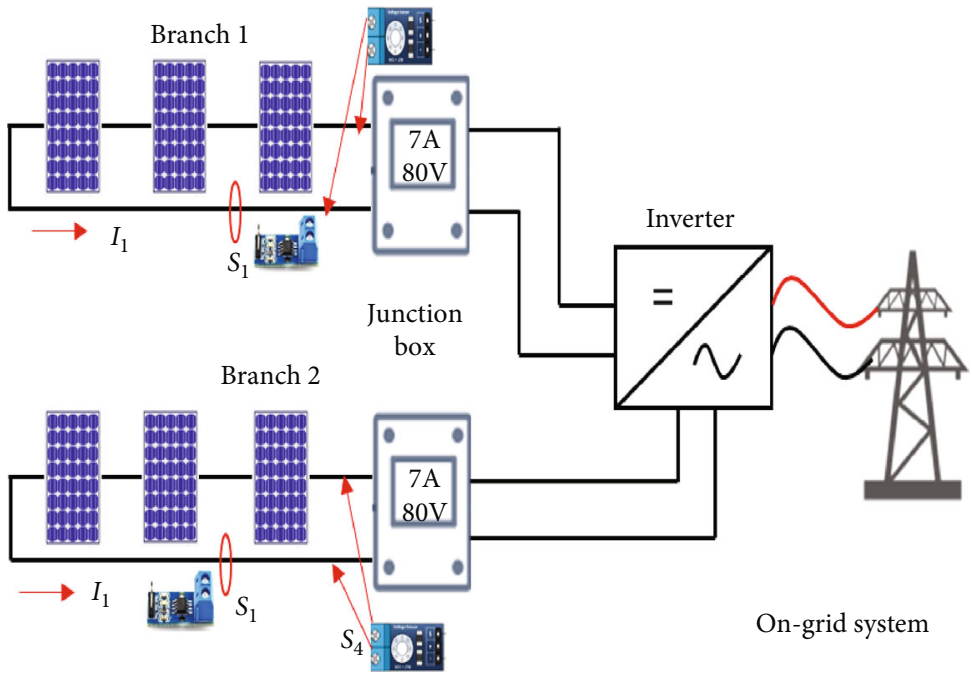

Figure 6: The experimental model of the PV system.

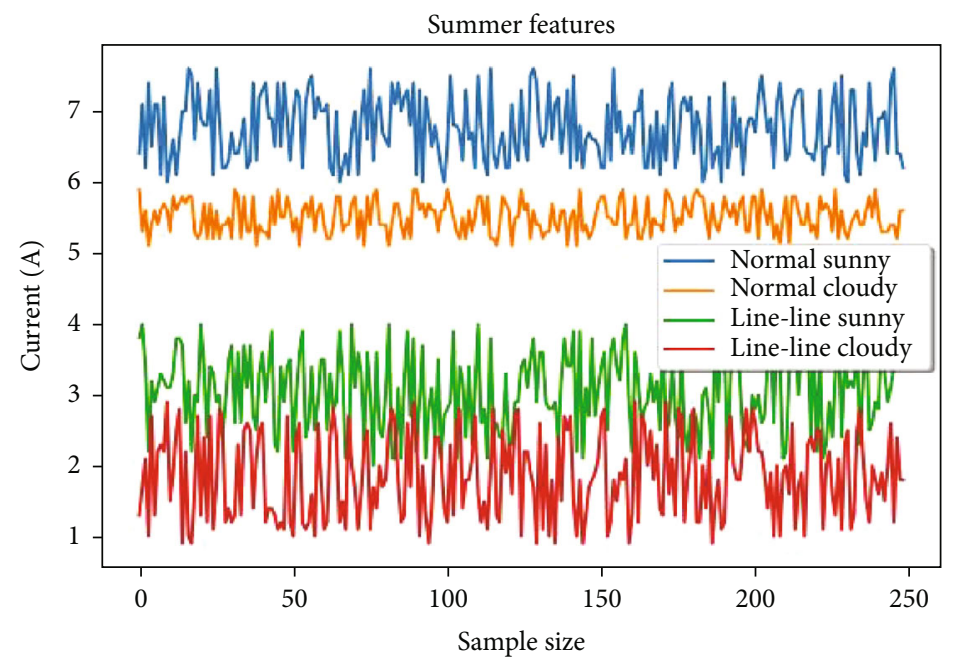

FIgURE 7: Normal and line-line fault dataset features during the summer season.

where $w_{k, j}^{N}$ is the weight between the neuron $k$ in the $N^{\text {th }}$ hidden layer and the neuron $j$ in the output layer and $n_{N}$ is the number of the neurons in the $N^{\text {th }}$ hidden layer. The output layer also uses uniform distribution as the kernel initializer, but unlike hidden layers, it uses Softmax as the activation function to represent the logits into probabilities [32]. The Softmax function is given as

$$
F\left(X_{i}\right)=\frac{\exp \left(X_{i}\right) i=0,1,2, \cdots k}{\sum_{j=0}^{k} \exp \left(x_{j}\right)} .
$$

Because of the nature of classification, we have used categorical crossentropy as the loss function given in equation (3) where $\hat{y}$ is the predicted output.

$$
L(y, \widehat{y})=-\sum_{j=0}^{M} \sum_{i=0}^{N}\left(y_{i j} * \log \left(\widehat{y}_{i j}\right)\right) .
$$

Categorical crossentropy will compare the distribution of the predictions (the activations in the output layer, one for each class) with the true distribution, where the probability of the true class is set to 1 and 0 for the other classes. Among many other optimizers, we used Adam (Adaptive Moment Estimation) for optimizing the proposed model [33]. Adam uses adaptive learning for each of the parameters, and the weight of a learning rate is divided by a running average of recent gradients. Finally, the model is fitted to train with a batch size of 5 with 200 epochs. Table 1 shows the different parameters used to construct the MLP as the fault classifier.

To check the bias-variance tradeoff, a $k$-fold crossvalidation test is performed with the 5 validations split into the training data. Also, for improving the model and reducing overfitting, we implemented the dropout regularization technique. The dropout rates of 0.1 and 0.2 were selected for the first and second hidden layers, respectively. The result of the evaluation, improvement, and tuning of the model is provided in Section 4.1. 


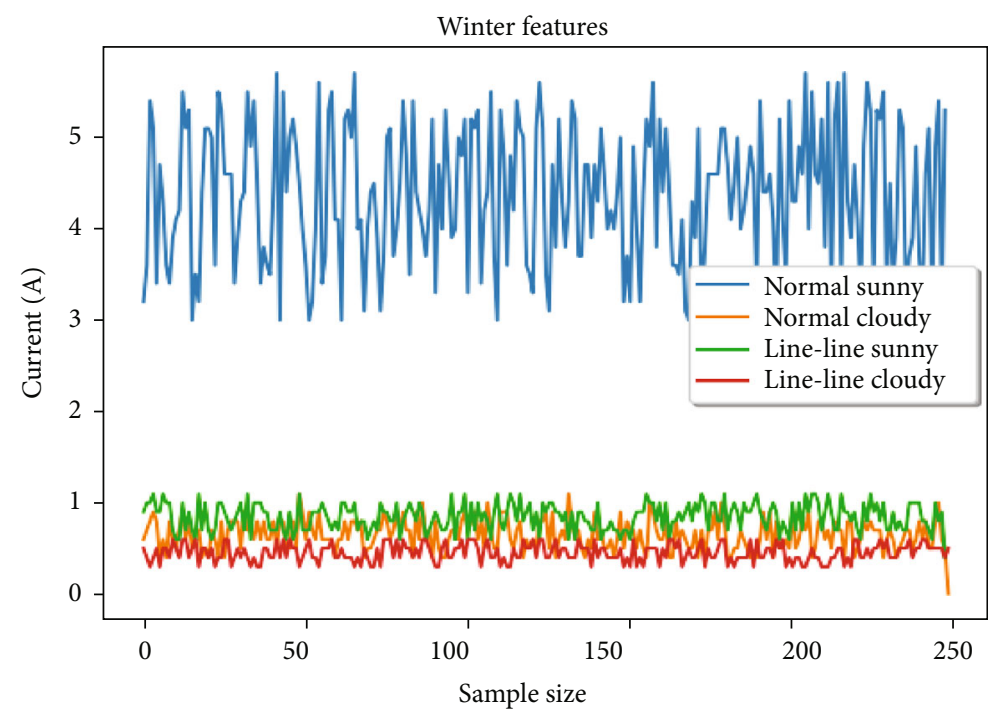

FIGURE 8: Normal and line-line fault dataset features during the winter season.

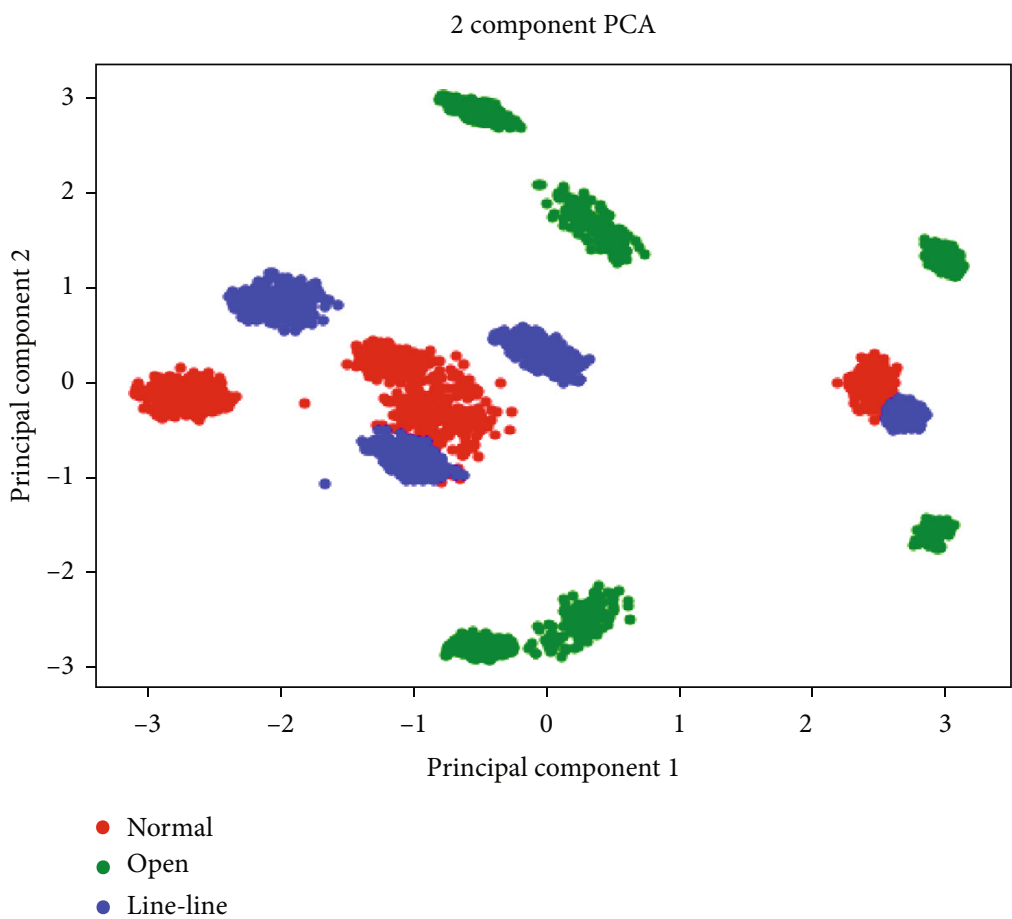

Figure 9: PCA dimension reduction technique for visualizing the multidimensional data.

\section{Results and Discussions}

4.1. Experimental Results. For the purpose of collecting experimental data, the data without any hardware or circuit modification in the PV system were categorized as "normal." Fault data were collected by making several intentional faults in the circuit of the PV array. Table 2 shows the minimum range, maximum range, and variance of data collected in the different environmental conditions to train the proposed model. For the experimental verification, we set up a PV system used in the real power pro- duction industry with the specifications given in Table 3 and Figure 6.

As shown in Table 2, variance in the winter dataset is very high than that in the summer season which requires special attention while training the model for accurate predictions.

Figures 7 and 8 show normal and line-line fault dataset features of the input variable $x_{1}$ and $x_{2}$ during summer and winter seasons during sunny days and cloudy days, respectively.

Among the input dataset, irradiation level seems to have the highest variance level in the absolute terms. However, visualizing the current sensor $\left(S_{I 1} / S_{I 2}\right)$ data would make 


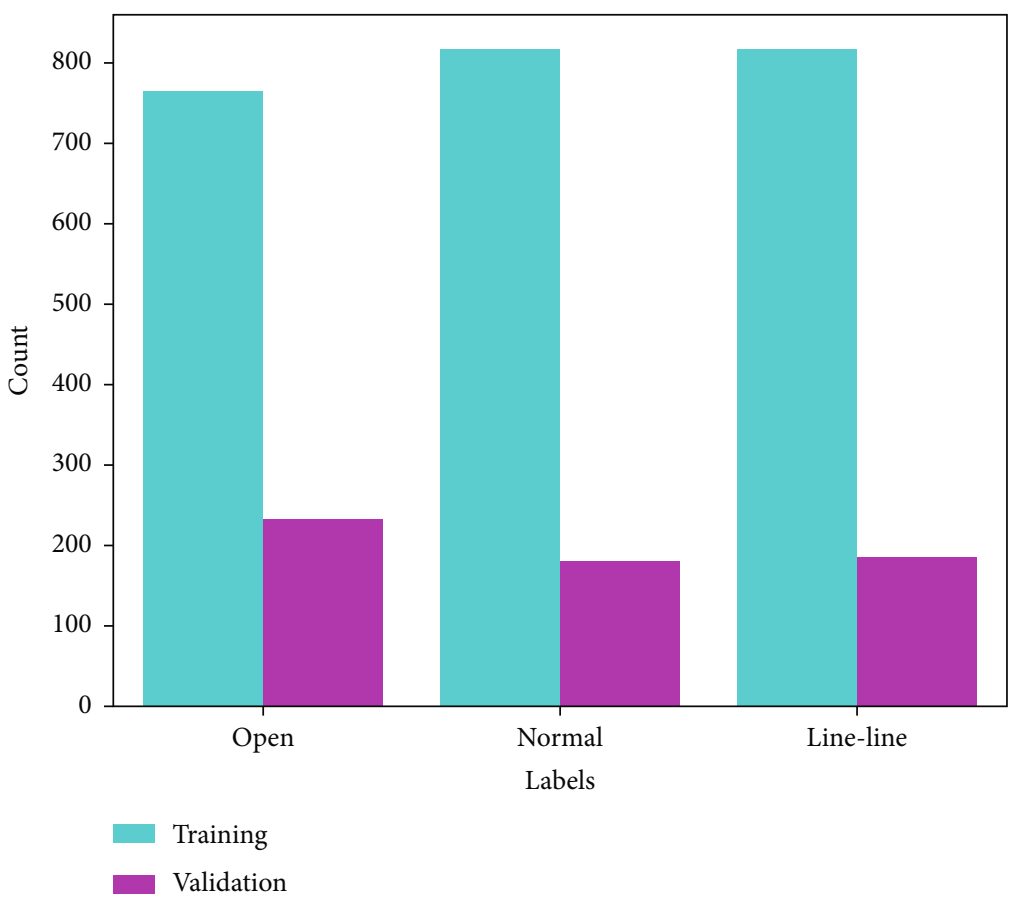

Figure 10: Training-test validation dataset of the collected data.

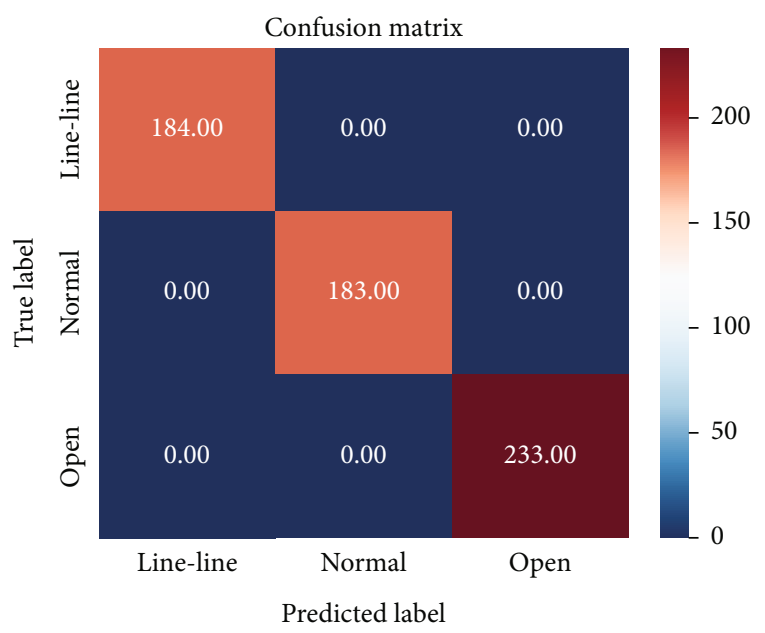

FIGURE 11: Confusion matrix for the true and predicted label data.

much sense since its relative variance $\left(\sigma_{x}^{2} / \bar{x}\right)$ was the highest among other input variables. As seen in Figure 7, it is hard to distinguish "normal cloudy" and "line-line" fault data in the winter season as they overlap most of the time.

Figure 9 shows the principal component analysis (PCA) dimensional reduction technique to visualize the sevendimension data $\left(x_{1}\right.$ to $\left.x_{7}\right)$ into scaled two-dimension data. As seen in the center-left and center-right parts of the figure, small regions exist where "normal" state data and "line-line" fault data overlap.

Figure 10 shows training-test validation of the dataset with the train-test split ratio of $8: 2$. The proposed PNN model was extensively trained with 3000 datasets, 1000 each from different states of the PV system.

Figure 11 shows the confusion matrix giving the outcome of $100 \%$ accuracy when the test data was fetched to the trained model. The numerical values shown in the confusion matrix are expressed in absolute terms, i.e., the total number of predicted labels for the line-line fault was 184, given the true label for the line-line fault was 184 , which is $100 \%$.

Figure 12 shows the setup of the experimental $1.8 \mathrm{~kW}$ PV system at the Jeonbuk National University campus.

Table 4 shows the comparison of the proposed method with existing studies found in the literature. Figure 13 shows the screenshot of the developed desktop application implementing the proposed model for fault detection.

4.2. Discussions. ML-based fault detection and diagnosis techniques have been employed recently, and it is expected to continue in the coming years. The quality of the MLbased model heavily depends upon the training data. Studies show the models trained with PV data tend to have very high accuracy rates in prediction, even up to $100 \%$ (Table 4). We tested our dataset with other machine learning models and got quite high accuracies (shown by the $F_{1}$ score) in each case as shown in Table 5 . The correlations between each predicted classifier are shown in Figure 14.

It is very important to identify the main features from the input dataset while training a ML model. The most important parameters in a PV system are current and voltage. A fault detection model only trained with these two input features can equally be robust as the other models trained with more input datasets.

No single fault detection technique is capable of detecting, diagnosing, and locating all types of faults in the PV 


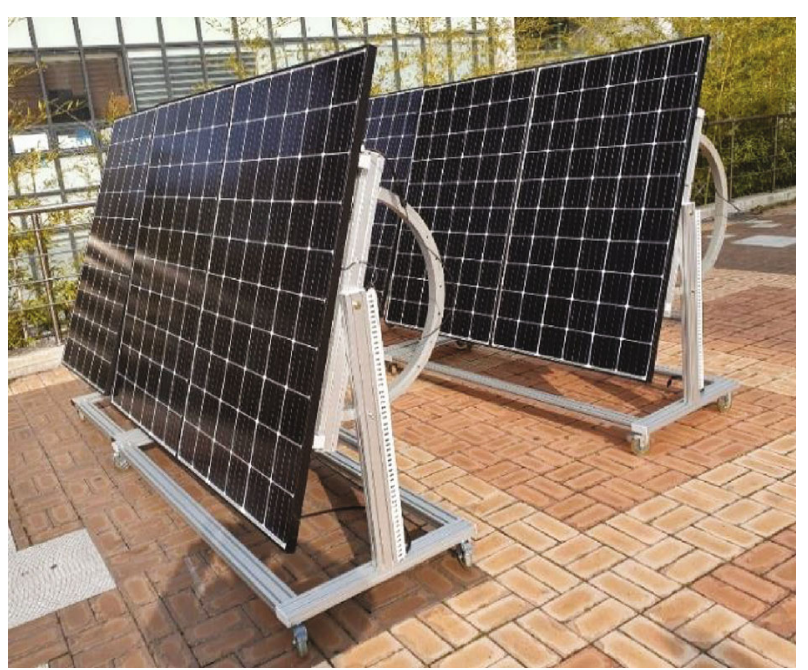

FIGURE 12: Setup of the experimental PV system at the premises of Jeonbuk National University.

TABle 4: Comparison with other models.

\begin{tabular}{lc}
\hline Model & Accuracy (\%) \\
\hline Li et al. [21] & 97 \\
Wua et al. [22] & 97.25 \\
Zhua et al. [23] & 100 \\
Sun et al. [24] & 97.5 \\
Kurukuru et al. [26] & 93.4 \\
Alajmi et al. [27] & 100 \\
Proposed & 100 \\
\hline
\end{tabular}

system. As discussed in the introduction part, our study is limited to detecting major electrical faults. As a part of the continuation of this study, we aim to continue the research to develop hybrid techniques including $\mathrm{ML}$ to develop a comprehensive and complete fault detection method.

In most of the cases, a fault detection model developed for a PV system cannot be implemented to another PV system as electrical parameters vary largely in different PV systems. There is a need for the development of flexible models that can be developed and can be implemented in any PV system with minor modifications. We have given special consideration to make our model as flexible as possible while developing the desktop application. The source code and data used for the experiment is available in the author's GitHub page as an open-source project. The open-source community can use the model for their application, provide feedback, or contribute to the improvement of the model as a whole.

\section{Conclusion}

PV systems are subject to various faults and failures, and early fault detection of those faults and failures is very impor-

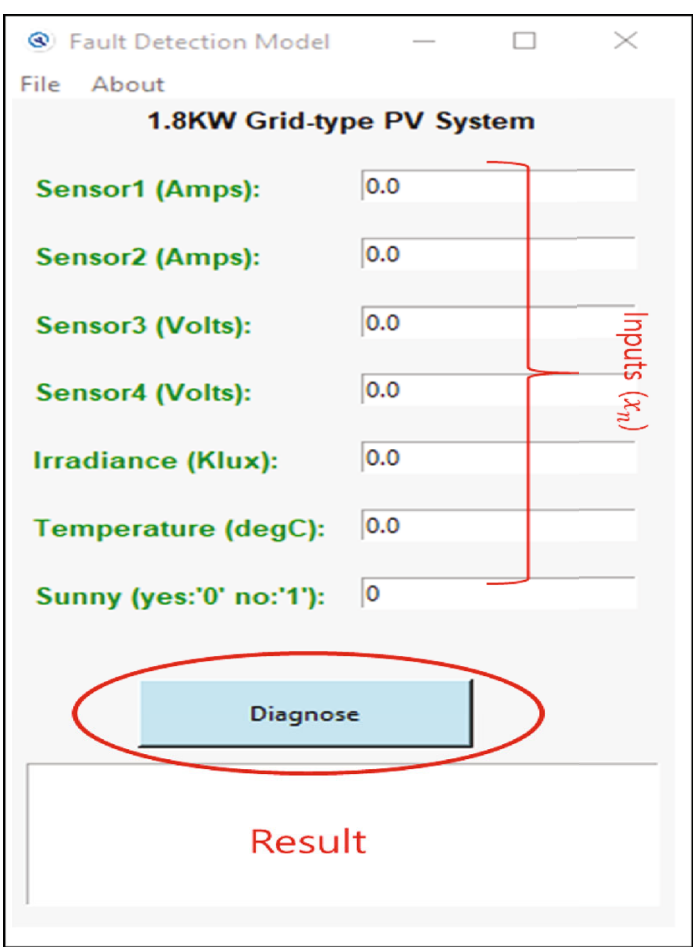

Figure 13: Screenshot of the developed desktop application implementing the proposed model.

TABLE 5: Other ML classifiers trained with the data.

\begin{tabular}{lc}
\hline Classifiers & $F_{1}$ score \\
\hline Support vector machine & 0.99 \\
$k$-Nearest neighbors & 1.0 \\
Random forest & 1.0 \\
Extra trees & 1.0 \\
AdaBoost & 0.85 \\
Naïve Bayes & 0.88 \\
\hline
\end{tabular}

tant for the efficiency and safety of the PV systems. ML-based fault detection models are trained with data and provide prediction results with very high accuracy. However, databased fault detection models for PV systems can sometimes give false predictions, especially when the environmental parameters are not taken into consideration. This paper developed an intelligent fault detection model for PV arrays based on PNN for accurately classifying the fault types. The model was trained with a large dataset containing different data values under different environmental conditions in the summer and the winter season. For the experimental verification, various fault state and normal state datasets are collected from $1.8 \mathrm{~kW}$ (six $300 \mathrm{~W}$ panels, 2 parallelly connected lines, each with 3 serially connected panels) into the grid-connected PV system. The experimental results demonstrate that the proposed method is superior in accurately predicting the result in cases where fault state and normal state are very hard to distinguish. 


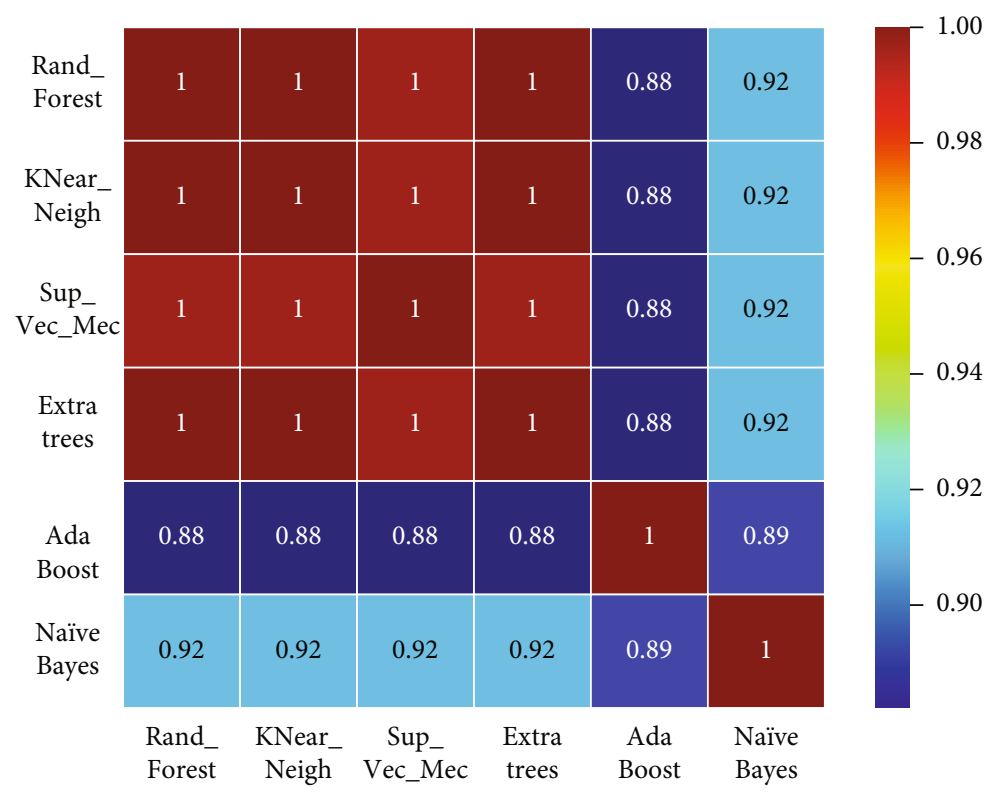

FIgURE 14: Correlations between predicted classifiers.

\section{Data Availability}

The source code and data used for the experiment are available in the author's GitHub page as an open-source project (http://github.com/benjamin2044/PV_fault_Python/tree/ master).

\section{Conflicts of Interest}

The authors declare that there are no conflicts of interest regarding the publication of this paper.

\section{Acknowledgments}

This research was supported by the Basic Science Research Program through the National Research Foundation of Korea (NRF) funded by the Ministry of Education (2018R1D1A1B07048630).

\section{References}

[1] M. Sabbaghpur Arani and M. A. Hejazi, "The comprehensive study of electrical faults in PV arrays," Journal of Electrical and Computer Engineering, vol. 2016, Article ID 8712960, 10 pages, 2016.

[2] A. Triki-Lahiani, A. Bennani-Ben Abdelghani, and I. SlamaBelkhodja, "Fault detection and monitoring systems for photovoltaic installations: a review," Renewable and Sustainable Energy Reviews, vol. 82, pp. 2680-2692, 2018.

[3] Y. Hu and W. Cao, Theoretical analysis and implementation of photovoltaic fault diagnosis, Intech, 2016.

[4] A. Y. Appiah, X. Zhang, B. B. K. Ayawli, and F. Kyeremeh, "Review and performance evaluation of photovoltaic array fault detection and diagnosis techniques," International Journal of Photoenergy, vol. 2019, Article ID 6953530, 19 pages, 2019.
[5] J. Dong, C. Zhang, M. Lv, Y. Ma, and N. Guan, "Photovoltaic array fault detection by automatic reconfiguration," Energies, vol. 10, no. 5, p. 699, 2017.

[6] D. S. Pillai and N. Rajesekar, "A comprehensive review on protection challenges and fault diagnosis in PV systems," Renewable and Sustainable Energy Reviews, vol. 91, pp. 18-40, 2018.

[7] A. Haque, K. V. S. Bharath, M. A. Khan, I. Khan, and Z. A. Jaffery, "Fault diagnosis of photovoltaic modules," Energy Science \& Engineering, vol. 7, pp. 622-644, 2019.

[8] M. H. Ali, A. Rabhi, A. El Hajjaji, and G. M. Tina, "Real time fault detection in photovoltaic systems," Energy Procedia, vol. 111, pp. 914-923, 2017.

[9] Y. Hu, W. Cao, J. Ma, S. Finney, and D. Li, "Identifying PV module mismatch faults by a thermography-based temperature distribution analysis," IEEE Transactions on Device and Materials Reliability, vol. 14, no. 4, pp. 951-960, 2014.

[10] M. Aghaei, U. E. Madukanya, A. K. V. de Oliveira, and R. Ruther, "Fault inspection by aerial infrared thermography in a PV plant after a meteorological tsunami," in VII Congresso Brasileiro de Energia Solar-Gramado, 17 a 20 de Abril de, Brazil, 2018.

[11] M. Muttilo, I. Nardi, V. Stornelli, T. de Rubeis, G. Pasqualoni, and D. Ambrosini, "On field infrared thermography sensing for PV system efficiency assessment: results and comparison with electrical models," Sensors, vol. 20, no. 4, p. 1055, 2020.

[12] L. Schirone, P. F. Califano, U. Moschella, and U. Rocca, "Fault finding in a $1 \mathrm{MW}$ photovoltaic plant by reflectometry," in Proceedings of 1994 IEEE 1st World Conference on Photovoltaic Energy Conversion - WCPEC (A Joint Conference of PVSC, PVSEC and PSEC), Waikoloa, HI, USA, 1994.

[13] T. Takashima, J. Yamaguchi, K. Otani, K. Kato, and M. Ishida, "Experimental studies of failure detection methods in PV module strings," in 2006 IEEE 4th World Conference on Photovoltaic Energy Conference, Waikoloa, HI, USA, 2006.

[14] S. Roy, M. K. Alam, F. Khan, J. Johnson, and J. Flicker, “An irradiance-independent, robust ground-fault detection scheme for PV arrays based on spread spectrum time-domain 
reflectometry (SSTDR)," IEEE Transactions on power electronics, vol. 33, no. 8, pp. 7046-7057, 2018.

[15] Z. Wang, S. McConnell, R. S. Balog, and J. Johnson, “Arc fault signal detection-Fourier transformation vs wavelet decomposition techniques using synthesized data," in 2014 IEEE 40th Photovoltaic Specialist Conference (PVSC), pp. 3239-3244, Denver, CO, USA, June 2014.

[16] Z. Wang and R. S. Balog, "Arc fault and flash detection in DC photovoltaic arrays using wavelets," in 2013 IEEE 39th Photovoltaic Specialists Conference (PVSC),, pp. 1619-1624, Tampa, FL, USA, June 2013.

[17] I. S. Kim, "Fault detection algorithm of the photovoltaic system using wavelet transform," in India International Conference on Power Electronics 2010 (IICPE2010), pp. 1-6, New Delhi, India, 2011.

[18] Z. Yi and A. H. Etemadi, "Line-to-line fault detection for photovoltaic arrays based on multiresolution signal decomposition and two-stage support vector machine," IEEE Transactions on Industrial Electronics, vol. 64, no. 11, pp. 8546-8556, 2017.

[19] M. Dhimish, V. Holmes, and M. Dales, "Parallel fault detection algorithm for grid-connected photovoltaic plants," Renewable Energy, vol. 113, pp. 94-111, 2017.

[20] D. S. Pillai, F. Blaabjerg, and N. Rajesekar, "A comparative evaluation of advanced fault detection approaches for PV systems," IEEE Journal of Photovoltaics, vol. 9, no. 2, pp. 513-527, 2019.

[21] Y. F. Li, P. J. Lin, H. F. Zhou et al., "On-line monitoring system of PV array based on internet of things technology," IOP Conference Series: Earth and Environmental Science, vol. 93, article 012078, 2017.

[22] Y. Wua, Z. Chena, L. Wua, P. Lina, S. Chenga, and P. Lu, "An intelligent fault diagnosis approach for PV array based on SARBF kernel extreme learning machine," Energy Procedia, vol. 105, pp. 1070-1076, 2017.

[23] H. Zhua, L. Lu, J. Yaoa, S. Dai, and Y. Hu, "Fault diagnosis approach for photovoltaic arrays based on unsupervised sample clustering and probabilistic neural network model," Solar Energy, vol. 176, pp. 395-405, 2018.

[24] J. Sun, F. Sun, J. Fan, and Y. Liang, "Fault diagnosis model of photovoltaic array based on least squares support vector machine in Bayesian framework," Applied Sciences, vol. 7, no. 11, article 1199, 2017.

[25] B. Ganeshprabu and M. Geethanjali, "Dynamic monitoring and optimization of fault diagnosis of photo voltaic solar power system using ANN and memetic algorithm," Circuits and Systems, vol. 7, no. 11, pp. 3531-3540, 2016.

[26] V. S. B. Kurukuru, A. Haque, M. A. Khan, and A. K. Tripathy, "Fault classification for photovoltaic modules using thermography and machine learning techniques2019 International Conference on Computer and Information Sciences (ICCIS),, Sakaka, Saudi Arabia, 2019.

[27] M. Alajmi, S. Aljahdali, S. Alsaheel, M. Fattah, and M. Alshehri, "Machine learning as an efficient diagnostic tool for fault detection and localization in solar photovoltaic arrays," in Proceedings of 32nd International Conference on Computer Applications in Industry and Engineering, vol. 63, pp. 21-33, USA, 2019.

[28] V. S. B. Kurukuru, F. Blaabjerg, M. A. Khan, and A. Haque, "A novel fault classification approach for photovoltaic systems," Energies, vol. 13, no. 2, p. 308, 2020.
[29] Y. Zhao, B. Lehman, J. F. DePalma, J. Mosesian, and R. Lyons, "Fault evolution in photovoltaic array during night-to-day transition," in 2010 IEEE 12th Workshop on Control and Modeling for Power Electronics (COMPEL), pp. 1-6, Boulder, CO, USA, 2010.

[30] Y. Zhao, B. Lehman, J. F. de Palma, J. Mosesian, and R. Lyons, "Fault analysis in solar PV arrays under: low irradiance conditions and reverse connections," in 2011 37th IEEE Photovoltaic Specialists Conference, Seattle, WA, USA, 2011.

[31] A. F. Agarap, "Deep learning using rectified linear units (ReLU)," 2019, https://arxiv.org/abs/1803.08375.

[32] C. E. Nwankpa, W. Ijomah, A. Gachagan, and S. Marshall, "Activation functions: comparison of trends in practice and research for deep learning," 2018, https://arxiv.org/abs/1811 .03378 .

[33] D. P. Kingma and J. Ba, "Adam: a method for stochastic optimization,” 2014, https://arxiv.org/abs/1412.6980. 